\title{
Effects of storage period on kinematic viscosity and density of biodiesel and its blends with ultra-low-sulfur diesel fuel at constant storage temperature
}

\author{
Youssef Kassem ${ }^{\mathrm{a}, \mathrm{b}}$, Berk Aktuğ ${ }^{\mathrm{a}}$, Elçin Özgenç ${ }^{\mathrm{a}}$, Mohammed S. Dib ${ }^{\mathrm{a}}$, Mohammed M. M. \\ Ghisheer $^{\mathrm{a}}$, Oluwole A. Cole ${ }^{\mathrm{a}}$ and Hüseyin Çamur ${ }^{\mathrm{a}}$ \\ a Department of Mechanical Engineering, Faculty of Engineering, Near East University, 99138, Nicosia, Cyprus \\ ${ }^{b}$ Department of Civil Engineering, Faculty of Engineering, Near East University, 99138, Nicosia, Cyprus
}

\begin{abstract}
In this experimental work, the viscosity and density of methyl ester based waste cooking vegetablebiodiesel oils were investigated under varying temperature and blend ratio of ultra-low sulfur diesel summer (ULSDS). The transesterified fatty acid methyl ester of waste cooking vegetable oils collected from local restaurants and cafes was used as neat biodiesel. Six different fuel blends $(5 \%, 10 \%, 15 \%$, $20 \%, 50 \%$ and $80 \%$ by volume mixing with base biodiesel) were studied along with base ULSDS fuel and pure biodiesel. Tests for viscosity and density were performed in the temperature range $-13^{\circ} \mathrm{C}-90^{\circ} \mathrm{C}$. It is found that pure biodiesel has the highest viscosity and density at a given temperature among biodiesel blends. Moreover, the density of each fuel sample decreases linearly with the increase in temperature. On the other hand, the viscosity decreases exponentially with the increasing temperature of each fuel sample. Moreover, the objective of this experimental work was to study the changes in the properties of biodiesel and the mixtures of the biodiesel with ULSDS during the long-term storage. The fuel samples were stored for 12 weeks at constant temperatures $\left(40^{\circ} \mathrm{C} \pm 1\right)$ and properties such as kinematic viscosity and density were periodically measured. The observations of the sample were tested every two weeks for a period of 12 weeks. Based on the experimental results, kinematic viscosity and density were increased upon extended storage by only small increments.
\end{abstract}

Keywords: Biodiesel, density, kinematic viscosity, storage period, ultra low sulfur diesel summer

\section{Introduction}

Biodiesel is a renewable and environmentally friendly alternative fuel, which is comprised of monoalkyl esters of long chain fatty acids derived from vegetable oils or animal fats [1]. Biodiesel and its blends with petroleum-based diesel fuel can be used in diesel engines without any significant modification to the engine [2-4]. The advantages of biodiesel over petroleum diesel are improved lubricity, a higher flash point, lower toxicity, and biodegradability [5-7]. Also, since biodiesel is oxygenated, combustion is more complete and produces fewer harmful emissions and pollutants [8,9].

Density and viscosity of biodiesel are two important physical properties because they are widely used in combustion models, design-operation-control of processes, and fuel quality. Viscosity, a measure of resistance to flow of a liquid due to the internal fluid friction [10,11], is an important property because of its direct relation with the fuel injection process in engines [11,12]. Biodiesel viscosity is usually higher than that of diesel, which results in longer liquid penetration and worse atomization [13, 14] compared with diesel fuel. Substance density is defined as its mass per unit volume. Density is a physical property that can be used to calculate the precise volume of fuel necessary to supply an adequate combustion. The

\footnotetext{
* Manuscript received January 7, 2018; revised April 12, 2018..

Corresponding author. E-mail address: yousseuf.kassem@neu.edu.tr.

doi: $10.12720 /$ sgce.7.2.130-144
} 
injection system, formed by high-pressure pump and injectors, enters to the cylinder a discrete volume of fuel, which is calculated by the Electronic Control Unit of the vehicle depending on the driving conditions [15]. The density of fuels mainly affects the spray momentum and the distribution of equivalence ratio. Apart of that, density is commonly used in numerous unit operations of biodiesel production [16]. Also, density values are required to properly design: reactors, distillation units, storage tanks, and pipes.

Biodiesel can be used as fuel or blended with petroleum in any percentage. However, the standard storage and handling procedures used for biodiesel are the main issue due to the biodiesel fuel specifications [17]. Several studies have shown that biodiesel storage duration and temperature are associated with adoption of biodiesel properties and quality of biodiesel.

Consequently, this study aimed to investigate the effects of storage duration of variant blending biodiesel ratio under constant storage temperature on fuel properties in terms of kinematic viscosity and density. The biodiesel samples were stored in laboratory oven at $40 \pm 1^{\circ} \mathrm{C}$ and were estimated at regular interval over a period of 12 weeks. The observations of the samples were tested every two weeks for a period of 12 weeks. Moreover, the effects of testing temperature on properties of biodiesel such as kinematic viscosity and density were examined. Furthermore, a graphic representation of viscosity and density as a simultaneous function of temperature and volume fraction as well as volume fraction and storage period in three-dimensional plots (3D-plots) is made

\section{Experimental Methods}

\subsection{Material}

The biodiesel used in this experimental work was transesterified fatty acid methyl ester of mixture of waste cooking oils and was collected from local cafes and restaurants. In this study, the termed pure biodiesel is denoted as B100 and was mixed with ultra low sulfur diesel summer (ULSDS). Therefore, the other fuel samples were termed as B5, B10, B15, B20, B50 and B80, i.e. 50\% of pure biodiesel is mixed with 50\% ULSDS. Biodiesel blends were prepared by weighting with an analytic balance. The uncertainty was $\pm 0.0001 \mathrm{~g}$. The system was mixed perfectly into a homogeneous solution by a magnetic stirrer before the experimental measurement of density and viscosity was done. The composition of the biodiesel with regard to fatty acid methyl ester was determined by gas chromatography (GC) in accordance with The American Oil Chemical Society official methods (AOCS). The results of GC and the fuel properties of biodiesel and ULSDS are presented in Table 1.

Table 1. Physico-chemical properties of biodiesel and ULSDS

\begin{tabular}{lllll}
\hline Property & Unit & Method & ULSDS & Biodiesel \\
\hline FAME content & mass\% & EN 14103 & - & $>99.5$ \\
Density at $\mathbf{1 5}^{\circ} \mathrm{C}$ & $\mathrm{kg} / \mathrm{m}^{3}$ & ASTM D 4052 & 827.8 & 860 \\
Cetan number & - & ASTM D 613 & 55 & 51.0 \\
Kinematic viscosity at $\mathbf{4 0}{ }^{\circ} \mathrm{C}$ & $\mathrm{mm}^{2} / \mathrm{s}$ & ASTM D 455 & 2.8 & 3.5 \\
CFPP & ${ }^{\circ} \mathrm{C}$ & IP 309 & -6 & 5 \\
Flash point & ${ }^{\circ} \mathrm{C}$ & ASTMD 93 & 67 & 101 \\
\hline Fatty acid Methyl ester & - & - & - & \\
- C12:0 & 0.3 & & & \\
C14:0 & 1.3 & & & \\
C16:0 & 21.4 & & & \\
C16:1 & 1.9 & & \\
C18:0 & 10.4 & & \\
C18:1 & 42.0 & & \\
C18:2 & 17.5 & & \\
C18:3 & 2.4 & & \\
C20:0 & 0.3 & & \\
C20:1 & 0.6 & & \\
\hline
\end{tabular}




\subsection{Storage test procedures}

$2000 \mathrm{~mL}$ sample was prepared for pure biodiesel and each blend and stored in closed glass bottles of $500 \mathrm{~mL}$ capacity for 12 weeks. The samples were kept indoors, at $40 \pm 1^{\circ} \mathrm{C}$ in the dark oven for which the temperature was controlled with a thermostat in the laboratory. Samples were taken out periodically every two weeks to study the storage condition effects.

\subsection{Viscosity and density measurements at various temperature from 20 to $90^{\circ} \mathrm{C}$}

Kinematic viscosity $\left(\mathrm{mm}^{2} / \mathrm{s}\right)$ was determined with Ubbelohde viscometer following the ASTM Standard D-445. The kinematic viscosity was measured in the temperature range $\mathbf{2 0}^{\circ} \mathrm{C}-\mathbf{9 0}^{\circ} \mathrm{C}$ with an accuracy of $\pm 0.1 \%$. Fig. 1 shows the experimental setup used to determine the temperature dependence of kinematic viscosity of the samples analyzed. To ensure precise and stable temperature control during measurements, a digital temperature controller resistance was used to monitor the temperature. A uniform temperature inside the heating bath was attained. In addition, the mixer enabled the regulation of the temperature of a heated oil bath containing the viscometer by means of an electric heater. Moreover, in order to ensure the temperature in heating bath is homogenous and constant, two $\mathrm{T}$ types thermocouples were used to measure the temperature in heating bath using multi-thermometer as shown in Fig. 1. Each sample was tested three times, and the average kinematic viscosity was calculated. In order to precisely determine the relationship between the time of flow and the kinematic viscosity for the two viscometers used, calibration of the instrument was necessary. The calibration was done by the manufacturer, SI Analytics GmbH, Mainz according to ASTM D 2525/ D 446 and ISO/DIS 3105. The instrument constant, $\mathrm{K},[(\mathrm{mm} 2 / \mathrm{s}) / \mathrm{s}]$ was determined and given as in Table 2 . The calibration constant can be used up to the temperature of $270^{\circ} \mathrm{C}$, and the influence of the temperature on the capillary constant due to thermal expansion of the Duran glass was negligible. For absolute measurement, the corrected flow time multiplied by the viscometer constant $\mathrm{K}$ directly gives the kinematic viscosity $\left[\mathrm{mm}^{2} / \mathrm{s}\right]$ as given in Equation (1).

$$
v=K(t-y)
$$

where $v, K$, t, and y represent the kinematic viscosity, the calibration constant, measured time of flow and kinetic energy correction, respectively.
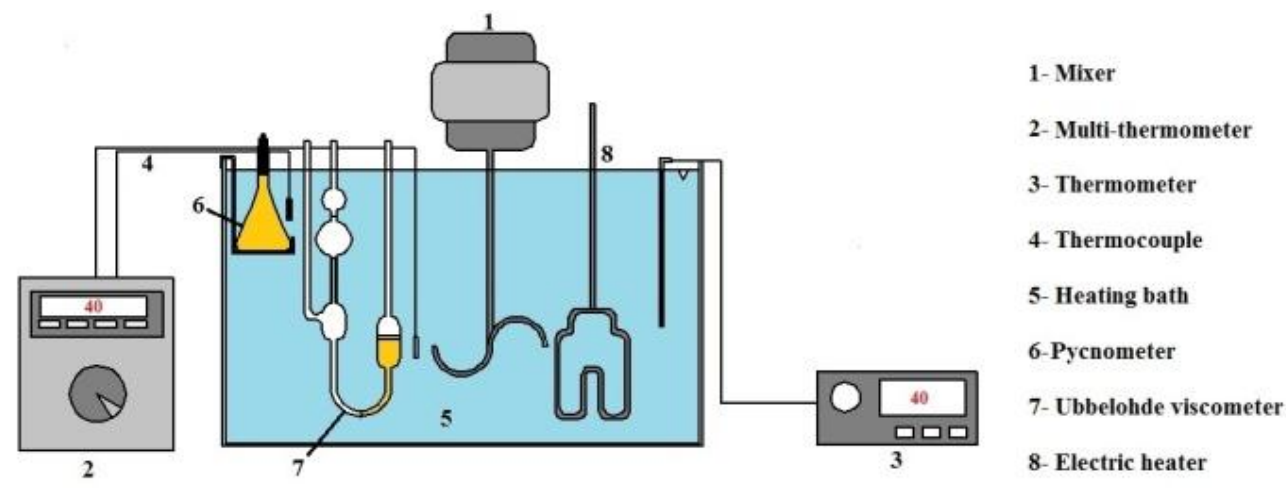

Fig. 1. Schematic of the experimental setup used to measure the viscosity and density of fuel samples in the temperature range 20$90^{\circ} \mathrm{C}$.

Table 2. Ubbelohde viscometer technical specifications

\begin{tabular}{cccc}
\hline Capillary No. & $\begin{array}{c}\text { Capillary } \\
\text { Dia. } \mathbf{I} \pm \mathbf{0 . 0 1}[\mathbf{m m}]\end{array}$ & $\begin{array}{c}\text { Constant }, \mathbf{K}, \\
\left(\mathbf{m m}^{2} / \mathbf{s}\right) / \mathbf{s}\end{array}$ & Measuring range $\left[\mathbf{m m}^{2} / \mathbf{s}\right]$ \\
\hline I & 0.58 & 0.009132 & 2 to 10 \\
Ic & 0.78 & 0.029440 & 6 to 30 \\
\hline
\end{tabular}


The density of the biodiesel was measured using a Pycnometer with a bulb capacity of $25 \mathrm{ml}$. The weighing was done by using a high precision electronic balance with a precision of $\pm 0.1 \mathrm{mg}$. The experimental setup of measuring the density of biodiesel samples from 20 to $90^{\circ} \mathrm{C}$ is shown in Fig. 1 . Each fuel was tested three times, and the average density was estimated.

\subsection{Viscosity and density measurements at various temperature from -13 to $20^{\circ} \mathrm{C}$}

Fig. 2 shows the experimental setup used to measure the kinematic viscosity and density of biodiesel and its blends with ULSDS in the temperature range -13 to $20^{\circ} \mathrm{C}$. The experimental setup consists of Ubbelohde viscometer, Pycnometer, a compressor, a mixer, multi-thermometer and a thermostat. Alcohol (ethanol) is the simplest and cheapest cooling bath. To obtain a uniform temperature distribution within cooling bath, the cooling bath is equipped with a mixer to circulate the alcohol. The bath temperature was controlled using a thermostat by automatically starting up and shutting down the compressor. Also, two T types thermocouples were used to measure the temperature in cooling bath using multi-thermometer at different locations. A coil connected to a compressor cools down the liquid bath and the compressor is cooled down by a radiator as shown in Fig. 2. The cooling bath was thermally isolated from the rest of its surroundings by a $3 \mathrm{~cm}$ thick Styrofoam layer.

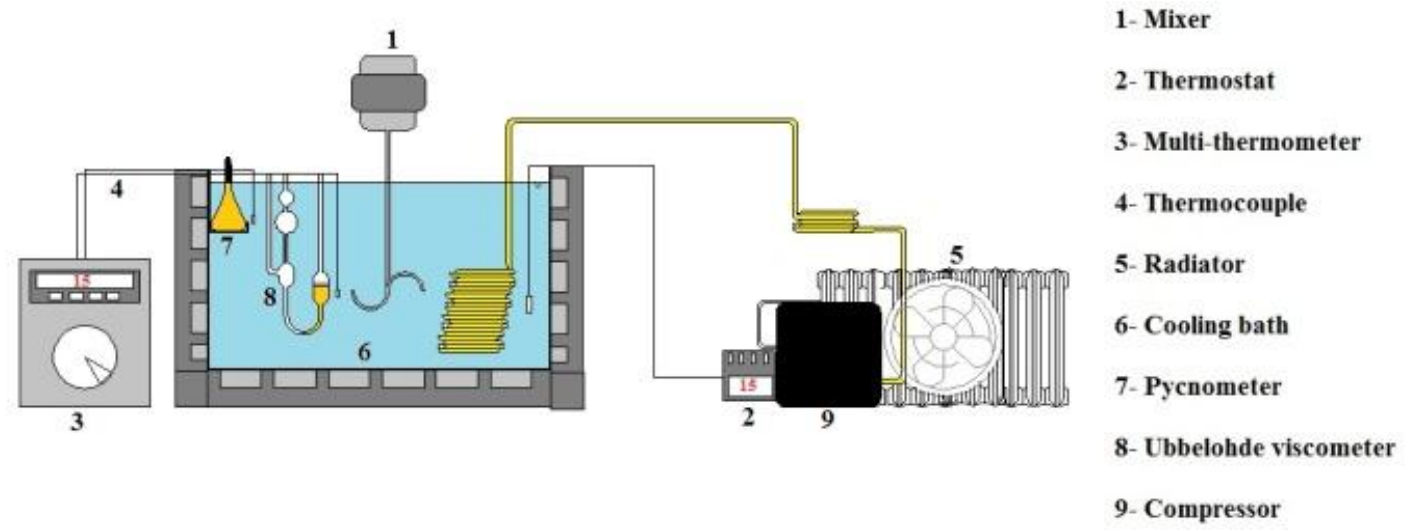

Fig. 2. Schematic of the experimental setup used to measure the viscosity and density of fuel samples in the temperature range -13$20^{\circ} \mathrm{C}$.

\subsection{Statistical analysis}

The experimental data obtained from this work were also analyzed by response surface methodology (RSM) by the response surface regression approach of second-order polynomial equation (Eq. (2)).

$$
Y=\beta_{0}+\sum_{i=1}^{n} \beta_{i} x_{i}+\sum_{i=1}^{n} \beta_{i i} x_{i}^{2}+\sum_{i}^{n-1} \sum_{i=i+1}^{n} \beta_{i j} x_{i} x_{j}
$$

where Y represents the predicted response; $\beta_{o}$ is the offset term; $\beta_{i}$ is the linear coefficient; the secondorder coefficient and $\beta_{i j}$ is the interaction coefficient; $x_{i}$ and $x_{j}$ are the independent variables. The method of least squares was employed to ascertain the values of the model parameters and analysis of variance (ANOVA) was applied to establish their statistical significance at a confidence level of $95 \%$.

\section{Results and Discussions}

\subsection{Effects of test temperature on kinematic viscosity of biodiesel blends ( 0 week)}

Fig. 3 shows the effects of testing temperature on kinematic viscosities of biodiesel blends. It can be seen that the kinematic viscosities of pure biodiesel are higher than ULSDS in the temperature range -13 $90^{\circ} \mathrm{C}$. The obtained kinematic viscosity data for biodiesel blends correspond with the recommended 
ASTM D455 values: $1.9-6 \mathrm{~mm}^{2} / \mathrm{s}$ at $40^{\circ} \mathrm{C}$. Moreover, it is found that the pure biodiesel (B100) offers the highest viscosity while the biodiesel-diesel (D100) has the lowest kinematic viscosity at a given temperature. It is also observed that with the increase of temperature, the viscosity of each fuel dropped exponentially as shown in Fig. 3.

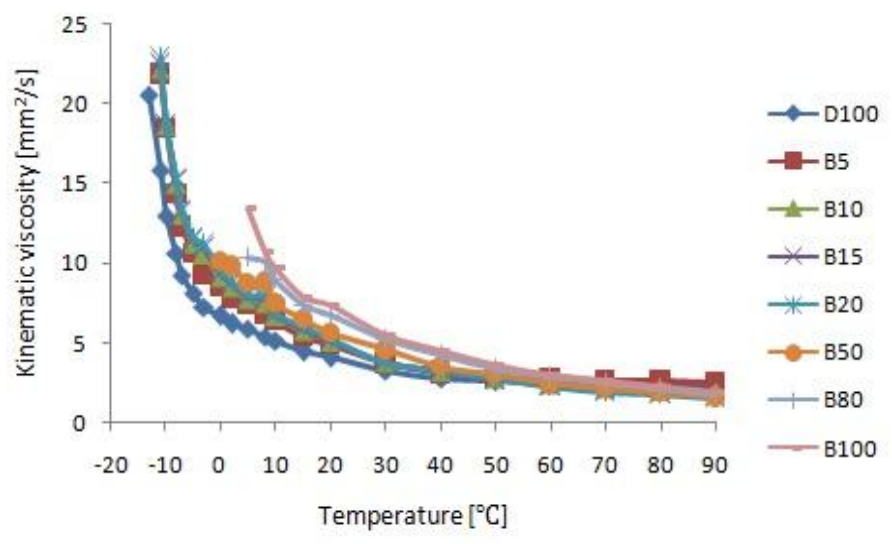

Fig. 3. Measured kinematic viscosity of all blends from -13 to $90^{\circ} \mathrm{C}$.

\subsection{Effects of test temperature on density of biodiesel blends ( 0 week)}

Fig. 4 shows the densities of waste cooking biodiesel (B100), B80, B50, B20, B15, B10, B5 and ULSDS (D100) from $-13^{\circ} \mathrm{C}$ to $90^{\circ} \mathrm{C}$ at atmospheric pressure. D100 has the lowest densities among the blends. The densities for all fuels decreased as the test temperature increased.

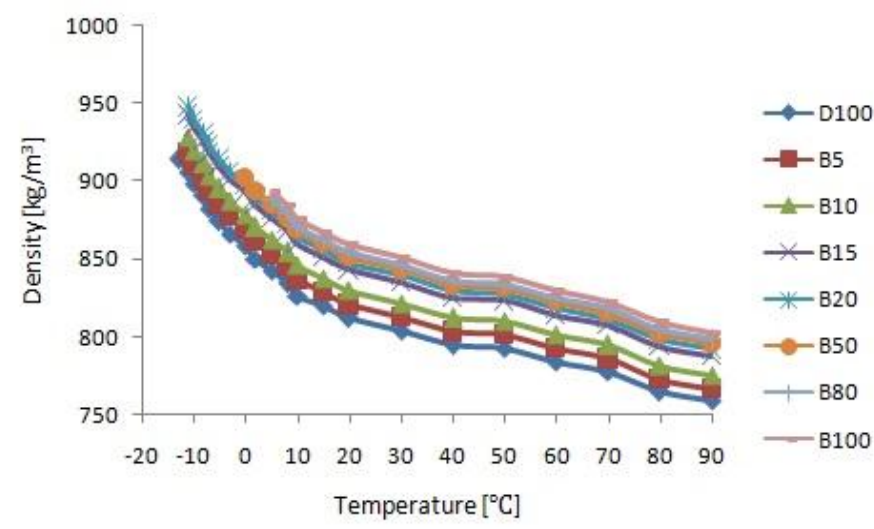

Fig. 4. Measured density of all blends from -13 to $90^{\circ} \mathrm{C}$

\subsection{Effects of storage period on kinematic viscosity of biodiesel blends}

The experimental data of kinematic viscosity (at test temperature $40^{\circ} \mathrm{C}$ ) for biodiesel samples for different storage periods are presented in Table 3. The initial kinematic viscosities of eight samples (Table 3) were within the specified ranges contained in ASTM D455. The kinematic viscosity of studied fuels increases with the increase of storage period as shown in Fig. 5 and Table 3. It is observed that the kinematic viscosity values of B5, B10, B15 and B20 over a storage period of 12 weeks are in accordance with a standard demand for diesel fuel (ASTM D 975 recommended values: $1.9-4.1 \mathrm{~mm}^{2} / \mathrm{s}$ at $40^{\circ} \mathrm{C}$ ) as shown in Fig. 5. While, after 10 weeks, the viscosity value of $\mathrm{B} 50$ at $40^{\circ} \mathrm{C}$ is higher than the specified ranges values of viscosity diesel fuel (ASTM D 975). 
The increase ratio $\left(\mathrm{I}_{\mathrm{r}}\right)$ is defined as the kinematic viscosity of a given sample at 12 weeks of storage divided by its initial kinematic viscosity at 0 week. Because kinematic viscosity of FAMEs increases with time, all $\mathrm{I}_{\mathrm{r}}$ values were greater than 1.00 as shown in Table 3.

Table 3. Influence of storage period (weeks) at constant storage temperature on kinematic viscosity $\left(40^{\circ} \mathrm{C}\right)$ in $\mathrm{mm}^{2} / \mathrm{s}$

\begin{tabular}{lrrrrrrrl}
\hline Weeks & $\mathbf{0}$ & $\mathbf{2}$ & $\mathbf{4}$ & $\mathbf{6}$ & $\mathbf{8}$ & $\mathbf{1 0}$ & $\mathbf{1 2}$ & $\mathbf{I}_{\mathbf{r}}$ \\
\hline D100 & 2.80 & 2.82 & 2.85 & 2.87 & 2.89 & 2.92 & 2.94 & 1.048 \\
B5 & 3.08 & 3.14 & 3.20 & 3.26 & 3.32 & 3.38 & 3.46 & 1.123 \\
B10 & 3.19 & 3.25 & 3.31 & 3.38 & 3.44 & 3.51 & 3.59 & 1.126 \\
B15 & 3.23 & 3.29 & 3.35 & 3.42 & 3.48 & 3.55 & 3.62 & 1.123 \\
B20 & 3.25 & 3.31 & 3.37 & 3.44 & 3.50 & 3.57 & 3.64 & 1.120 \\
B50 & 3.48 & 3.65 & 3.74 & 3.86 & 3.92 & 4.09 & 4.17 & 1.198 \\
B80 & 4.19 & 4.28 & 4.33 & 4.41 & 4.45 & 4.54 & 4.58 & 1.094 \\
B100 & 4.54 & 4.60 & 4.63 & 4.68 & 4.72 & 4.76 & 4.79 & 1.054 \\
\hline
\end{tabular}

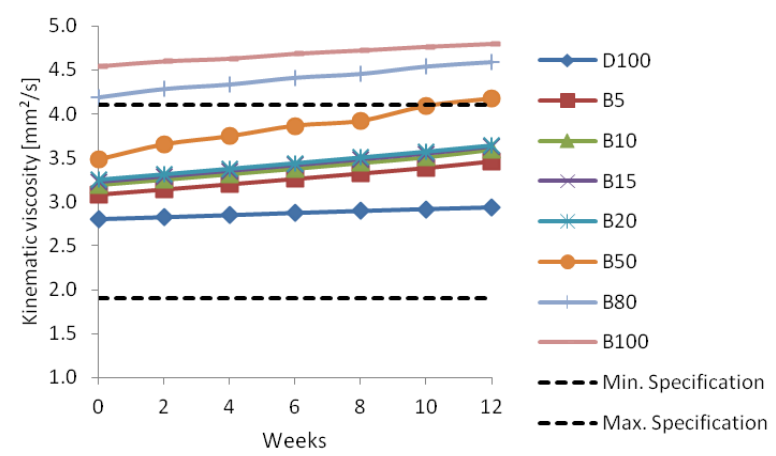

Fig. 5. Measured viscosity of all blends at test temperature $40^{\circ} \mathrm{C}$.

In addition, the influence of testing temperature on kinematic viscosity for some selected storage periods has been tabulated in Table 4 . It is observed that the samples demonstrate temperature-dependent behaviour and the kinematic viscosity is increasing over the storage period for the same testing temperature. An increase of the storage period leads to increase the kinematic viscosity of biodiesel blends. Fig. 6 shows the relationship between the kinematic viscosities and the temperature for a storage period of 6 weeks. The kinematic viscosity decreases nonlinearly with increasing temperature.

Table 4. Influence of storage periods and testing temperatures on kinematic viscosity in $\mathrm{mm}^{2} / \mathrm{s}$ of blends

\begin{tabular}{|c|c|c|c|c|c|c|c|c|c|c|c|c|c|c|c|c|c|}
\hline \multirow{2}{*}{$\begin{array}{c}\mathbf{T} \\
{\left[{ }^{\circ} \mathbf{c}\right.} \\
]\end{array}$} & \multicolumn{8}{|c|}{ 0 Week } & \multirow{2}{*}{$\begin{array}{c}\mathbf{T} \\
{\left[{ }^{\circ} \mathbf{c}\right.} \\
]\end{array}$} & \multicolumn{8}{|c|}{6 Week } \\
\hline & $\begin{array}{c}\text { D10 } \\
\text { 0 }\end{array}$ & B5 & B10 & B15 & B20 & B50 & B80 & $\begin{array}{c}\text { B10 } \\
\text { 0 }\end{array}$ & & $\begin{array}{c}\text { D10 } \\
\text { 0 }\end{array}$ & B5 & B10 & B15 & B20 & B50 & B80 & $\begin{array}{c}\text { B10 } \\
\text { 0 }\end{array}$ \\
\hline- & $\begin{array}{c}20.6 \\
1\end{array}$ & nd & nd & nd & $\mathrm{Nd}$ & nd & nd & nd & - & 20.7 & nd & nd & nd & nd & nd & nd & nd \\
\hline 13 & $\begin{array}{c}1 \\
158\end{array}$ & & & & & & & & 13 & $\begin{array}{c}7 \\
159\end{array}$ & & 236 & 234 & 234 & nd & nd & $d$ \\
\hline - & $\begin{array}{c}15.8 \\
5\end{array}$ & $\begin{array}{c}21.8 \\
9\end{array}$ & $\begin{array}{c}22.1 \\
7\end{array}$ & $\begin{array}{c}22.4 \\
9\end{array}$ & $\begin{array}{c}22.8 \\
9\end{array}$ & nd & nd & nd & $\overline{11}$ & $\begin{array}{c}15.9 \\
8\end{array}$ & $\begin{array}{c}24.1 \\
7\end{array}$ & $\begin{array}{c}25.0 \\
5\end{array}$ & $\begin{array}{c}23.4 \\
8\end{array}$ & 23.4 & nd & nd & nd \\
\hline- & 12.9 & 18.4 & 18.6 & 18.7 & 18.7 & nd & nd & nd & - & 13.0 & 18.8 & 19.0 & 19.1 & 19.1 & nd & nd & nd \\
\hline 10 & 7 & 7 & 5 & 1 & 3 & & & & 10 & 8 & 1 & 3 & 1 & 5 & & & \\
\hline-8 & $\begin{array}{c}10.6 \\
2\end{array}$ & $\begin{array}{c}14.3 \\
7\end{array}$ & $\begin{array}{c}15.0 \\
1\end{array}$ & $\begin{array}{c}15.2 \\
2\end{array}$ & $\begin{array}{c}15.3 \\
3\end{array}$ & nd & nd & nd & -8 & 10.7 & $\begin{array}{c}14.6 \\
4\end{array}$ & $\begin{array}{c}15.3 \\
2\end{array}$ & $\begin{array}{c}15.5 \\
5\end{array}$ & $\begin{array}{c}15.6 \\
7\end{array}$ & nd & nd & nd \\
\hline-7 & 9.27 & $\begin{array}{c}12.2 \\
9\end{array}$ & $\begin{array}{c}13.0 \\
2\end{array}$ & $\begin{array}{c}13.2 \\
7\end{array}$ & $\begin{array}{c}13.3 \\
9\end{array}$ & nd & nd & nd & -7 & 9.35 & $\begin{array}{c}12.5 \\
2\end{array}$ & 13.3 & $\begin{array}{c}13.5 \\
5\end{array}$ & $\begin{array}{c}13.6 \\
8\end{array}$ & nd & nd & nd \\
\hline-5 & 8.16 & $\begin{array}{c}10.6 \\
1\end{array}$ & $\begin{array}{c}11.2 \\
5\end{array}$ & $\begin{array}{c}11.4 \\
6\end{array}$ & $\begin{array}{c}11.5 \\
6\end{array}$ & nd & nd & nd & -5 & 8.23 & $\begin{array}{c}11.0 \\
2\end{array}$ & $\begin{array}{c}11.5 \\
5\end{array}$ & $\begin{array}{c}11.7 \\
3\end{array}$ & $\begin{array}{c}11.8 \\
2\end{array}$ & nd & nd & nd \\
\hline-3 & 7.23 & 9.24 & $\begin{array}{c}10.5 \\
3\end{array}$ & $\begin{array}{c}10.9 \\
6\end{array}$ & $\begin{array}{c}11.1 \\
8\end{array}$ & nd & nd & nd & -3 & 7.29 & 9.7 & $\begin{array}{c}10.8 \\
6\end{array}$ & $\begin{array}{c}11.2 \\
4\end{array}$ & $\begin{array}{c}11.4 \\
4\end{array}$ & nd & nd & nd \\
\hline $\mathbf{0}$ & 6.68 & 8.49 & 9.15 & 9.37 & 9.48 & $\begin{array}{c}10.1 \\
1\end{array}$ & nd & nd & $\mathbf{0}$ & 6.74 & 8.54 & 9.2 & 9.42 & 9.53 & $\begin{array}{c}10.2 \\
2\end{array}$ & nd & nd \\
\hline 2 & 6.18 & 7.8 & 8.49 & 8.72 & 8.83 & 9.79 & nd & nd & 2 & 6.23 & 7.93 & 8.59 & 8.81 & 8.92 & 9.97 & nd & nd \\
\hline 5 & 5.86 & 7.37 & 7.78 & 7.91 & 7.98 & 8.74 & $\begin{array}{c}10.3 \\
3\end{array}$ & $\begin{array}{c}13.5 \\
2\end{array}$ & 5 & 5.91 & 7.83 & 8.02 & 8.08 & 8.11 & 8.89 & $\begin{array}{c}10.4 \\
5\end{array}$ & nd \\
\hline
\end{tabular}




\begin{tabular}{|c|c|c|c|c|c|c|c|c|c|c|c|c|c|c|c|c|c|}
\hline 8 & 5.43 & 6.79 & 7.53 & 7.78 & 7.91 & 8.8 & $\begin{array}{c}10.0 \\
4\end{array}$ & $\begin{array}{c}10.6 \\
6\end{array}$ & 8 & 5.47 & 6.89 & 7.66 & 7.91 & 8.04 & 8.08 & $\begin{array}{c}10.1 \\
7\end{array}$ & $\begin{array}{c}11.0 \\
7\end{array}$ \\
\hline 10 & 5.14 & 6.4 & 6.69 & 6.78 & 6.83 & 7.54 & 8.97 & 9.69 & 10 & 5.18 & 6.51 & 6.79 & 6.89 & 6.94 & 7.59 & 9.18 & 9.97 \\
\hline 15 & 4.43 & 5.39 & 5.78 & 5.91 & 5.97 & 6.49 & 7.4 & 7.86 & 15 & 4.47 & 5.47 & 5.87 & 6 & 6.07 & 6.55 & 7.77 & 8.38 \\
\hline 20 & 4.09 & 4.93 & 5.1 & 5.15 & 5.18 & 5.67 & 6.76 & 7.3 & 20 & 4.12 & 5.01 & 5.18 & 5.24 & 5.26 & 5.72 & 6.88 & 7.45 \\
\hline 30 & 3.28 & 3.72 & 3.76 & 3.77 & 3.77 & 4.57 & 5.2 & 5.52 & 30 & 3.31 & 3.78 & 3.82 & 3.83 & 3.83 & 4.76 & 5.46 & 5.8 \\
\hline 40 & 2.8 & 3.08 & 3.19 & 3.23 & 3.25 & 3.48 & 4.19 & 4.54 & 40 & 2.87 & 3.26 & 3.38 & 3.42 & 3.44 & 3.86 & 4.41 & 4.68 \\
\hline 50 & 2.59 & 2.85 & 2.75 & 2.71 & 2.69 & 3.05 & 3.39 & 3.56 & 50 & 2.61 & 3.02 & 2.91 & 2.87 & 2.85 & 3.12 & 3.55 & 3.77 \\
\hline 60 & 2.49 & 2.74 & 2.42 & 2.31 & 2.25 & 2.47 & 2.85 & 3.03 & 60 & 2.51 & 2.9 & 2.56 & 2.44 & 2.38 & 2.72 & 3.08 & 3.26 \\
\hline 70 & 2.44 & 2.69 & 2.18 & 2.01 & 1.93 & 2.17 & 2.48 & 2.64 & 70 & 2.46 & 2.84 & 2.31 & 2.13 & 2.04 & 2.33 & 2.67 & 2.84 \\
\hline 80 & 2.37 & 2.61 & 2.03 & 1.83 & 1.74 & 1.92 & 2.14 & 2.25 & 80 & 2.39 & 2.76 & 2.14 & 1.94 & 1.84 & 2.04 & 2.34 & 2.49 \\
\hline 90 & 2.3 & 2.54 & 1.89 & 1.67 & 1.56 & 1.61 & 1.78 & 1.86 & 90 & 2.32 & 2.69 & 2 & 1.77 & 1.65 & 1.77 & 2.07 & 2.21 \\
\hline $\mathbf{T}$ & \multicolumn{8}{|c|}{12 Weeks } & $\mathbf{T}$ & \multicolumn{8}{|c|}{12 Weeks } \\
\hline $\begin{array}{c}{\left[{ }^{\circ} \mathbf{c}\right.} \\
]\end{array}$ & $\begin{array}{c}\text { D10 } \\
\text { 0 }\end{array}$ & B5 & B10 & B15 & B20 & B50 & B80 & $\begin{array}{c}\text { B10 } \\
\text { 0 }\end{array}$ & $\begin{array}{c}{\left[{ }^{\circ} \mathbf{c}\right.} \\
]\end{array}$ & $\begin{array}{c}\text { D10 } \\
\text { 0 }\end{array}$ & B5 & B10 & B15 & B20 & B50 & B80 & $\begin{array}{c}\text { B10 } \\
\text { 0 }\end{array}$ \\
\hline$\overline{13}$ & $\begin{array}{c}20.9 \\
4\end{array}$ & nd & nd & nd & nd & nd & nd & nd & 10 & 5.22 & 6.62 & 7.41 & 7.68 & 7.81 & 7.64 & 9.57 & $\begin{array}{c}10.5 \\
3\end{array}$ \\
\hline$\overline{11}$ & $\begin{array}{c}16.1 \\
1\end{array}$ & nd & nd & nd & nd & nd & nd & nd & 15 & 4.5 & 5.57 & 6.44 & 6.73 & 6.88 & 6.56 & 7.82 & 8.45 \\
\hline- & $\begin{array}{c}13.1 \\
8\end{array}$ & $\begin{array}{c}19.3 \\
2\end{array}$ & $\begin{array}{c}21.5 \\
2\end{array}$ & nd & nd & nd & nd & nd & 20 & 4.15 & 5.09 & 6.16 & 6.51 & 6.69 & 5.76 & 6.94 & 7.54 \\
\hline-8 & $\begin{array}{c}10.7 \\
9\end{array}$ & $\begin{array}{c}15.0 \\
3\end{array}$ & $\begin{array}{c}16.7 \\
8\end{array}$ & $\begin{array}{c}17.3 \\
6\end{array}$ & $\begin{array}{c}17.6 \\
5\end{array}$ & nd & nd & nd & 30 & 3.34 & 3.85 & 4.19 & 4.31 & 4.37 & 4.81 & 5.58 & 5.97 \\
\hline-7 & 9.42 & $\begin{array}{c}12.8 \\
5\end{array}$ & $\begin{array}{c}14.6 \\
5\end{array}$ & $\begin{array}{c}15.2 \\
5\end{array}$ & $\begin{array}{c}15.5 \\
5\end{array}$ & nd & nd & nd & 40 & 2.94 & 3.45 & 3.57 & 3.61 & 3.64 & 4.17 & 4.58 & 4.79 \\
\hline-5 & 8.29 & $\begin{array}{c}11.3 \\
1\end{array}$ & $\begin{array}{c}11.9 \\
1\end{array}$ & $\begin{array}{c}12.1 \\
1\end{array}$ & $\begin{array}{c}12.2 \\
1\end{array}$ & nd & nd & nd & 50 & 2.64 & 3.07 & 3.23 & 3.29 & 3.31 & 3.16 & 3.55 & 3.75 \\
\hline-3 & 7.34 & $\begin{array}{c}10.2 \\
4\end{array}$ & 12.2 & $\begin{array}{c}12.8 \\
5\end{array}$ & $\begin{array}{c}13.1 \\
7\end{array}$ & nd & nd & nd & 60 & 2.53 & 2.95 & 2.8 & 2.75 & 2.73 & 2.76 & 3.1 & 3.28 \\
\hline 0 & 6.79 & 8.92 & 9.76 & $\begin{array}{c}10.0 \\
4\end{array}$ & $\begin{array}{c}10.1 \\
8\end{array}$ & $\begin{array}{c}10.3 \\
9\end{array}$ & nd & nd & 70 & 2.48 & 2.89 & 2.46 & 2.32 & 2.25 & 2.43 & 2.72 & 2.87 \\
\hline 2 & 6.28 & 8.2 & 9.25 & 9.6 & 9.78 & 9.99 & nd & nd & 80 & 2.41 & 2.8 & 2.28 & 2.11 & 2.02 & 2.09 & 2.4 & 2.56 \\
\hline 5 & 5.95 & 7.96 & 8.11 & 8.16 & 8.18 & 8.92 & nd & nd & 90 & 2.34 & 2.74 & 2.12 & 1.92 & 1.82 & 1.84 & 2.12 & 2.25 \\
\hline 8 & 5.51 & 7.01 & 8.43 & 8.9 & 9.13 & 8.09 & $\begin{array}{c}10.4 \\
9\end{array}$ & $\begin{array}{c}11.6 \\
9\end{array}$ & & & & & & & & & \\
\hline
\end{tabular}

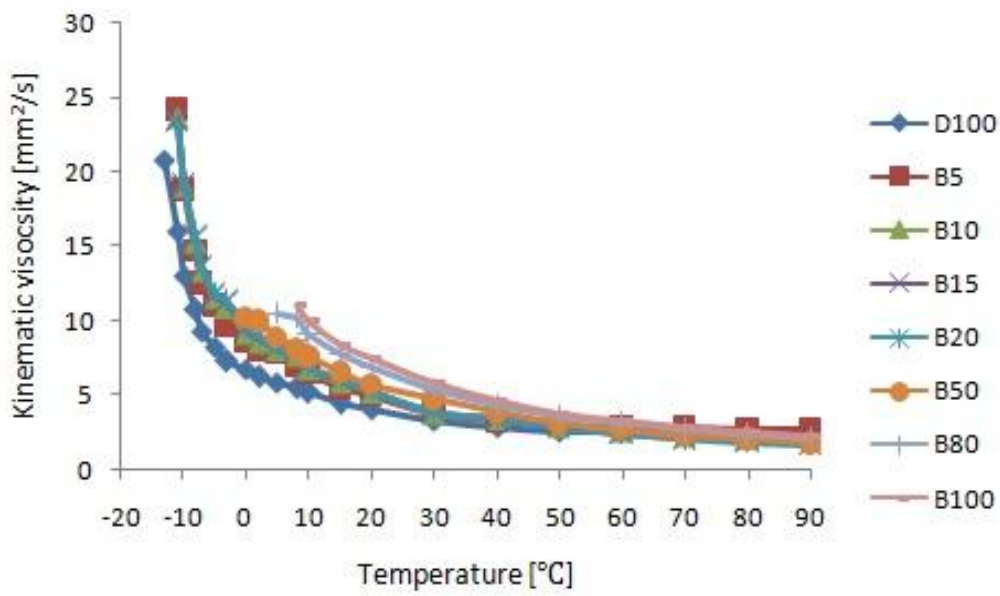

Fig. 6. Measured kinematic viscosity of all blends from -13 to $90^{\circ} \mathrm{C}$ over storage period of 6 weeks. 


\subsection{Effects of storage period on density of biodiesel blends}

The experimental results of higher density at testing temperature of $15^{\circ} \mathrm{C}$ for waste cooking oil methyl ester biodiesel and its blends with diesel at different storage period has been summarized in Table 5 .

Table 5. Influence of storage period (weeks) at constant storage temperature on density $\left(15^{\circ} \mathrm{C}\right) \mathrm{in} \mathrm{kg} / \mathrm{m}^{3}$

\begin{tabular}{lllllllll}
\hline Week & 0 & 2 & 4 & 6 & 8 & 10 & 12 & I $_{\mathbf{r}}$ \\
\hline D100 & 820.0 & 822.1 & 823.9 & 825.8 & 827.4 & 829.2 & 831.2 & 1.01 \\
B5 & 828.7 & 830.5 & 832.4 & 834.2 & 836.0 & 837.9 & 839.6 & 1.01 \\
B10 & 837.9 & 841.6 & 843.4 & 848.1 & 852.0 & 855.5 & 858.2 & 1.02 \\
B15 & 851.3 & 853.5 & 855.3 & 857.4 & 859.0 & 860.9 & 863.0 & 1.01 \\
B20 & 855.8 & 858.0 & 861.9 & 865.0 & 866.6 & 868.5 & 870.6 & 1.02 \\
B50 & 859.9 & 862.9 & 864.7 & 867.5 & 871.5 & 873.2 & 875.0 & 1.02 \\
B80 & 862.6 & 865.8 & 868.7 & 871.8 & 874.4 & 876.3 & 879.4 & 1.02 \\
B100 & 867.8 & 873.5 & 875.4 & 878.7 & 881.2 & 883.5 & 886.4 & 1.02 \\
\hline
\end{tabular}

Fig. 7 illustrates density values at $15^{\circ} \mathrm{C}$ for different biodiesel blends with ULSDS at different storage periods. It is observed that if the experimental results of biodiesel blends with ULSDS compared with specification diesel fuel standard (ASTM D4052) range between 800 and $880 \mathrm{~kg} / \mathrm{m}^{3}$ overall density results meet the standard or specification requirement of diesel fuel as shown in Fig. 7 and Table 5.

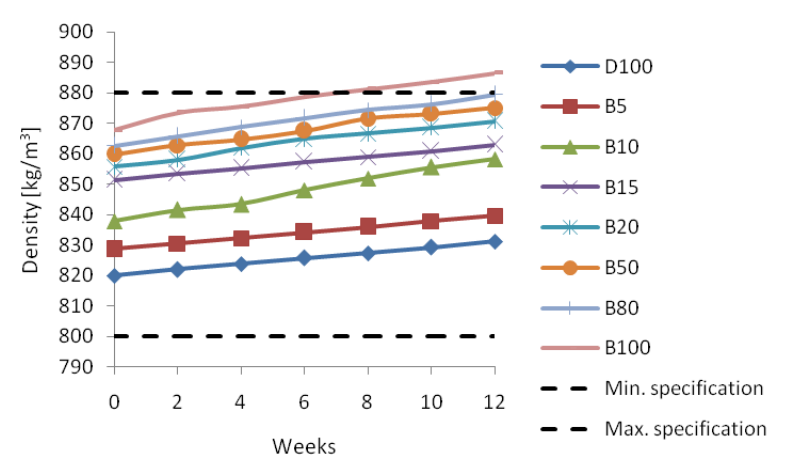

Fig. 7. Measured density of all blends at test temperature $15^{\circ} \mathrm{C}$.

The influence of testing temperature on density for some selected storage periods has been tabulated in Table 6. It is observed that the samples demonstrate temperature-dependent behaviour and the density is increasing over the storage period for the same testing temperature. An increase of the storage period leads to increase the density of biodiesel blends.

Table 6. Influence of storage periods and testing temperatures on density in $\mathrm{kg} / \mathrm{m}^{3}$ of blends

\begin{tabular}{|c|c|c|c|c|c|c|c|c|c|c|c|c|c|c|c|c|c|}
\hline \multirow{2}{*}{$\begin{array}{l}\mathbf{T} \\
{\left[{ }^{\circ}\right.} \\
\mathbf{c}]\end{array}$} & \multicolumn{8}{|c|}{ o Week } & \multirow{2}{*}{$\begin{array}{l}\text { T } \\
{\left[^{\circ}\right.} \\
\text { c] }\end{array}$} & \multicolumn{8}{|c|}{6 Week } \\
\hline & $\begin{array}{c}\text { D10 } \\
\text { 0 }\end{array}$ & B5 & B10 & B15 & B20 & B50 & B80 & $\begin{array}{c}\text { B10 } \\
\text { 0 }\end{array}$ & & $\begin{array}{c}\text { D10 } \\
\text { 0 }\end{array}$ & B5 & B10 & B15 & B20 & B50 & B80 & $\begin{array}{c}\text { B10 } \\
\text { 0 }\end{array}$ \\
\hline $\begin{array}{l} \\
13\end{array}$ & $\begin{array}{l}914 \\
.1\end{array}$ & nd & nd & nd & nd & nd & nd & nd & $\begin{array}{l}- \\
13\end{array}$ & $\begin{array}{l}916 \\
.8\end{array}$ & nd & nd & nd & nd & nd & nd & nd \\
\hline- & 906 & 917 & 928 & 942 & 947 & nd & nd & nd & - & 909 & 920 & 939 & 949 & 958 & nd & nd & nd \\
\hline 11 & .4 & .8 & .0 & .9 & .8 & & & & 11 & .1 & .6 & .3 & .6 & .0 & & & \\
\hline- & 898 & 909 & 919 & 934 & 939 & nd & nd & nd & - & 901 & 912 & 931 & 941 & 949 & nd & nd & nd \\
\hline 10 & .4 & .7 & .8 & .5 & .5 & & & & 10 & .1 & .4 & .0 & .2 & .5 & & & \\
\hline-8 & $\begin{array}{l}890 \\
.4\end{array}$ & $\begin{array}{l}901 \\
.6\end{array}$ & $\begin{array}{l}911 \\
.6\end{array}$ & $\begin{array}{l}926 \\
.2\end{array}$ & $\begin{array}{l}931 \\
.1\end{array}$ & nd & nd & nd & -8 & $\begin{array}{l}893 \\
.0\end{array}$ & $\begin{array}{l}904 \\
.3\end{array}$ & $\begin{array}{l}922 \\
.7\end{array}$ & $\begin{array}{l}932 \\
.8\end{array}$ & $\begin{array}{l}941 \\
.1\end{array}$ & nd & nd & nd \\
\hline-7 & $\begin{array}{l}882 \\
.3\end{array}$ & $\begin{array}{l}893 \\
.5\end{array}$ & $\begin{array}{l}903 \\
.4\end{array}$ & $\begin{array}{l}917 \\
.9\end{array}$ & $\begin{array}{l}922 \\
.7\end{array}$ & nd & nd & nd & -7 & $\begin{array}{l}885 \\
.0\end{array}$ & $\begin{array}{l}896 \\
.2\end{array}$ & $\begin{array}{l}914 \\
.4\end{array}$ & $\begin{array}{l}924 \\
.4\end{array}$ & $\begin{array}{l}932 \\
.6\end{array}$ & nd & nd & nd \\
\hline
\end{tabular}




\begin{tabular}{|c|c|c|c|c|c|c|c|c|c|c|c|c|c|c|c|c|c|}
\hline \multirow{2}{*}{-5} & 874 & 885 & 895 & 909 & 914 & nd & nd & nd & \multirow{2}{*}{-5} & 877 & 888 & 906 & 916 & 924 & nd & \multirow[t]{2}{*}{ nd } & \multirow[t]{2}{*}{ nd } \\
\hline & .3 & .4 & .2 & .5 & .3 & & & & & .0 & .0 & .1 & .0 & .1 & & & \\
\hline \multirow{2}{*}{-3} & 866 & 877 & 887 & 901 & 906 & nd & nd & nd & \multirow{2}{*}{-3} & 868 & 879 & 897 & 907 & 915 & nd & nd & nd \\
\hline & .3 & .3 & .0 & .2 & .0 & & & & & .9 & .9 & .8 & .6 & .7 & & & \\
\hline \multirow[t]{2}{*}{ 0 } & 858 & 869 & 878 & 892 & 897 & 901 & nd & nd & \multirow{2}{*}{ 0 } & 860 & 871 & 889 & 899 & 907 & 909 & nd & nd \\
\hline & .3 & .2 & .8 & .9 & .6 & .8 & & & & .9 & .8 & .5 & .2 & .2 & .8 & & \\
\hline \multirow[b]{2}{*}{2} & 850 & 861 & 870 & 884 & 889 & 893 & nd & nd & \multirow{2}{*}{2} & 852 & 863 & 881 & 890 & 898 & 901 & nd & nd \\
\hline & .3 & .1 & .6 & .6 & .2 & .4 & & & & .9 & .6 & .2 & .9 & .7 & .3 & & \\
\hline \multirow{2}{*}{5} & 842 & 853 & 862 & 876 & 880 & 885 & 887 & 893 & \multirow{2}{*}{5} & 844 & 855 & 872 & 882 & 890 & 892 & nd & nd \\
\hline & .3 & .0 & .4 & .2 & .9 & .0 & .9 & .2 & & .8 & .5 & .9 & .5 & .3 & .8 & & \\
\hline 8 & 834 & 844 & 854 & 867 & 872 & 876 & 879 & 884 & 8 & 836 & 847 & 864 & 874 & 881 & 884 & 888 & 903 \\
\hline 0 & .3 & .8 & .2 & .9 & .5 & .6 & .4 & .7 & 0 & .8 & .4 & .6 & .1 & .8 & .4 & .8 & .7 \\
\hline 10 & 826 & 836 & 846 & 859 & 864 & 868 & 871 & 876 & 10 & 828 & 839 & 856 & 865 & 873 & 875 & 880 & 895 \\
\hline 10 & .3 & .7 & .0 & .6 & .1 & .2 & .0 & .2 & 10 & .8 & .2 & .3 & .7 & .4 & .9 & .2 & .0 \\
\hline & 820 & 828 & 837 & 851 & 855 & 859 & 862 & 867 & & 825 & 834 & 848 & 857 & 865 & 867 & 871 & 886 \\
\hline 15 & .0 & .7 & .9 & .3 & .8 & .9 & .6 & .8 & 15 & .8 & .2 & .1 & .4 & .0 & .5 & .8 & .4 \\
\hline & 812 & 820 & 829 & 843 & 847 & 851 & 854 & 859 & & 814 & 823 & 839 & 849 & 856 & 859 & 863 & 877 \\
\hline 20 & .1 & .7 & .8 & .1 & .5 & .5 & .3 & .4 & 20 & .5 & .1 & .9 & .1 & .6 & .1 & .3 & .8 \\
\hline & 804 & 812 & 821 & 834 & 839 & 843 & 845 & 851 & & 806 & 815 & 831 & 840 & 848 & 850 & 854 & 869 \\
\hline 30 & .1 & .7 & .7 & .8 & .2 & .2 & .9 & .0 & 30 & .5 & .1 & .6 & .8 & .2 & .7 & .9 & .2 \\
\hline & 794 & 803 & 812 & 825 & 829 & 833 & 835 & 840 & & 797 & 805 & 821 & 830 & 838 & 840 & 844 & 859 \\
\hline 40 & .6 & .0 & .0 & .0 & .3 & .2 & .9 & .9 & 40 & .0 & .5 & .8 & .8 & .2 & .6 & .8 & .0 \\
\hline & 792 & 801 & 810 & 823 & 827 & 831 & 834 & 839 & & 795 & 803 & 819 & 828 & 836 & 838 & 842 & 857 \\
\hline 50 & .8 & .2 & .1 & .1 & .4 & .3 & .0 & .0 & 50 & .2 & .6 & .9 & .9 & .3 & .7 & .8 & .0 \\
\hline & 784 & 792 & 801 & 814 & 818 & 822 & 824 & 829 & & 786 & 794 & 810 & 819 & 827 & 829 & 833 & 847 \\
\hline 60 & .1 & .4 & .2 & .0 & .3 & .2 & .8 & .7 & 60 & .4 & .8 & .9 & .8 & .1 & .4 & .6 & .5 \\
\hline 70 & 777 & 785 & 794 & 807 & 811 & 815 & 818 & 822 & 70 & 779 & 788 & 804 & 813 & 820 & 822 & 826 & 840 \\
\hline 10 & .6 & .8 & .5 & .3 & .5 & .4 & .0 & .9 & 10 & .9 & .2 & .2 & .0 & .2 & .6 & .7 & .5 \\
\hline & 764 & 772 & 781 & 793 & 798 & 801 & 804 & 809 & 80 & 767 & 775 & 790 & 799 & 806 & 809 & 813 & 826 \\
\hline 80 & .7 & .8 & .4 & .9 & .1 & .9 & .5 & .3 & 80 & .0 & .2 & .9 & .6 & .7 & .0 & .0 & .6 \\
\hline & 758 & 766 & 775 & 787 & 791 & 795 & 798 & 802 & & 761 & 769 & 784 & 793 & 800 & 802 & 806 & 820 \\
\hline 90 & .7 & .8 & .3 & .7 & .9 & .6 & .2 & .9 & 90 & .0 & .1 & .7 & .3 & .4 & .7 & .7 & .2 \\
\hline $\mathbf{T}$ & & & & 12 & eeks & & & & $\mathbf{T}$ & & & & 12 & eeks & & & \\
\hline c] & $\begin{array}{c}\text { D10 } \\
\text { 0 }\end{array}$ & B5 & B10 & B15 & B20 & B50 & B80 & $\begin{array}{c}\text { B10 } \\
\text { 0 }\end{array}$ & c] & $\begin{array}{c}\text { D10 } \\
\text { 0 }\end{array}$ & B5 & B10 & B15 & B20 & B50 & B80 & $\begin{array}{c}\text { B10 } \\
\text { 0 }\end{array}$ \\
\hline - & 922 & & nd & nd & nd & nd & nd & nd & & 834 & 844 & 866 & 871 & 879 & 883 & 887 & 895 \\
\hline 13 & .8 & nd & & & & & & & 10 & .2 & .7 & .6 & .3 & .0 & .5 & .9 & .0 \\
\hline - & 915 & 926 & nd & nd & nd & nd & nd & nd & 15 & 831 & 839 & 858 & 863 & 870 & 875 & 879 & 886 \\
\hline 11 & .0 & .5 & & & & & & & 15 & .2 & .6 & .2 & .0 & .6 & .0 & .4 & .4 \\
\hline - & 906 & 918 & 942 & nd & nd & nd & nd & nd & & 819 & 828 & 849 & 854 & 862 & 866 & 870 & 877 \\
\hline 10 & .9 & .4 & .1 & & & & & & 20 & .8 & .5 & .9 & .6 & .2 & .5 & .9 & .8 \\
\hline-8 & 898 & 910 & 933 & 938 & 947 & nd & nd & nd & 30 & 811 & 820 & 841 & 846 & 853 & 858 & 862 & 869 \\
\hline -8 & .8 & .2 & .7 & .9 & .2 & & & & 30 & .8 & .4 & .6 & .2 & .7 & .1 & .4 & .2 \\
\hline & 890 & 902 & 925 & 930 & 938 & nd & nd & nd & & 802 & 810 & 831 & 836 & 843 & 847 & 852 & 859 \\
\hline-7 & .7 & .0 & .3 & .4 & .7 & & & & 40 & .2 & .7 & .7 & .2 & .6 & .9 & .2 & .0 \\
\hline & 882 & 893 & 916 & 922 & 930 & nd & nd & nd & & 800 & 808 & 829 & 834 & 841 & 846 & 850 & 857 \\
\hline-5 & .7 & .8 & .9 & .0 & .1 & & & & 50 & .3 & .8 & .8 & .3 & .7 & .0 & .2 & .0 \\
\hline-3 & 874 & 885 & 908 & 913 & 921 & nd & nd & nd & 6 & 791 & 799 & 820 & 825 & 832 & 836 & 840 & 847 \\
\hline-3 & .6 & .6 & .5 & .5 & .6 & & & & 60 & .5 & .9 & .6 & .1 & .4 & .7 & .9 & .5 \\
\hline 0 & 866 & 877 & 900 & 905 & 913 & 917 & nd & nd & 70 & 785 & 793 & 813 & 818 & 825 & 829 & 833 & 840 \\
\hline 0 & .5 & .4 & .1 & .1 & .1 & .7 & & & 70 & .0 & .3 & .8 & .3 & .5 & .7 & .9 & .5 \\
\hline 2 & 858 & 869 & 891 & 896 & 904 & 909 & nd & nd & 80 & 772 & 780 & 800 & 804 & 811 & 816 & 820 & 826 \\
\hline 2 & .4 & .3 & .8 & .6 & .6 & .2 & & & 80 & .0 & .2 & .4 & .8 & .9 & .0 & .1 & .6 \\
\hline 5 & 850 & 861 & 883 & 888 & 896 & 900 & nd & nd & 90 & 766 & 774 & 794 & 798 & 805 & 809 & 813 & 820 \\
\hline 5 & .3 & .1 & .4 & .2 & .1 & .6 & & & 90 & .0 & .1 & .1 & .5 & .6 & .6 & .7 & .2 \\
\hline 8 & 842 & 852 & 875 & 879 & 887 & 892 & 896 & 903 & & & & & & & & & \\
\hline ठ & .3 & .9 & .0 & .8 & .6 & .1 & .5 & .7 & & & & & & & & & \\
\hline
\end{tabular}

Moreover, Fig. 8 is an example to show the effect of the testing temperatures over storage period (12 weeks) for all blends. It is noticed that as the testing temperature increases, the density of the blends decreases. Also, storage over an extended period (12 weeks) resulted in higher density for all fuels. 


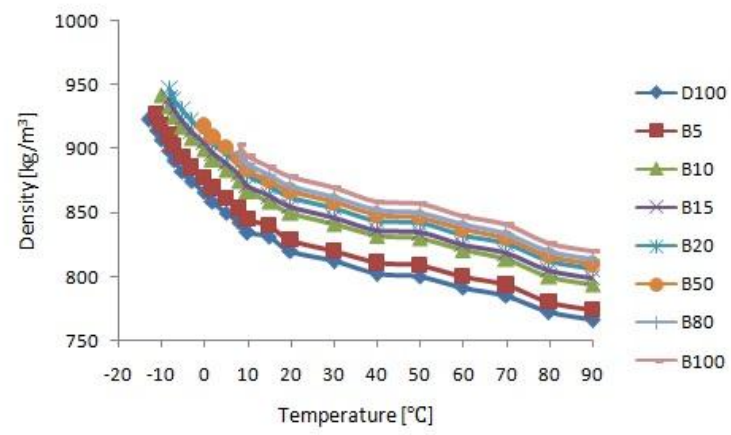

Fig. 8. Measured density of all blends from -13 to $90^{\circ} \mathrm{C}$ over storage period of 12 weeks.

\subsection{Optimization of viscosity and density of biodiesel blends}

The influence of testing temperatures, volume fraction (VF) of biodiesel and storage periods (W) on the viscosity $(v)$ and density $(\rho)$ of biodiesel blends was tested using RSM.

The experimental data were randomized to minimize the effects of unexpected variability in the observed responses. The methodology adopted allows the formulation of a second-order equation which describes the process. The properties of biodiesel blends were analyzed through the least squares method to fit the second order equation as shown in Table 7.

To test the fit of the models, the determination coefficient $\left(\mathrm{R}^{2}\right)$ was evaluated. Consequently, it follows that prediction results using RSM are in very good agreement with the actual values of the kinematic viscosity and density of biodiesel. This observation can be confirmed with very high value of coefficient of determination. For example, $\mathrm{R}^{2}$ of Eq. (3) was 0.833 , which indicated that the model could explain $83.30 \%$ of the variability. The $\mathrm{R}^{2}$ values showed that the experimental data for kinematic viscosity and density have a very good correlation with the models for all biodiesel. Therefore, $\mathrm{R}^{2}$ values are close to unity highlighting proper fitting of the predicted values.

Table 7. Correlations for calculating the viscosity and density of biodiesel blends

\begin{tabular}{|c|c|c|}
\hline Equations & \multirow{2}{*}{$\begin{array}{c}\mathbf{R}^{2} \\
{[\%]}\end{array}$} & \multirow{2}{*}{$\begin{array}{c}\text { Eq } \\
\dot{\text { No }}\end{array}$} \\
\hline$-13^{\circ} \mathrm{C} \leq \mathrm{T} \leq 90^{\circ} \mathrm{C}$ & & \\
\hline$v=10.319+3.696 . \mathrm{VF}-0.3336 . \mathrm{T}+0.002905 . \mathrm{VF}^{2}-0.044 . \mathrm{VF} . \mathrm{T}$ & 83.30 & 3 \\
\hline $20^{\circ} \mathrm{C} \leq \mathrm{T} \leq 90^{\circ} \mathrm{C}$ & \multirow[b]{2}{*}{96.61} & \multirow[b]{2}{*}{4} \\
\hline$v=6.643+2.221 . \mathrm{VF}-0.11904 . \mathrm{T}+1.148 \mathrm{VF}^{2}+0.00079 . \mathrm{T}^{2}-0.0433 . \mathrm{VF} . \mathrm{T}$ & & \\
\hline $13^{\circ} \mathrm{C} \leq \mathrm{T} \leq 20^{\circ} \mathrm{C}$ & \multirow[b]{2}{*}{89.03} & \multirow[b]{2}{*}{5} \\
\hline$v=7.836+9.62 . \mathrm{VF}-0.552 . \mathrm{T}+0.02347 . \mathrm{VF}^{2}-0.455 . \mathrm{VF} . \mathrm{T}$ & & \\
\hline $40^{\circ} \mathrm{C}$ & \multirow[b]{2}{*}{96.18} & \multirow[b]{2}{*}{6} \\
\hline$v=2.9180+0.0311 \mathrm{~W}+1.5907 . \mathrm{VF}$ & & \\
\hline$-13^{\circ} \mathrm{C} \leq \mathrm{T} \leq 90^{\circ} \mathrm{C}$ & \multirow{3}{*}{93.66} & \multirow[b]{2}{*}{7} \\
\hline$\rho=870.14+127.8 . \mathrm{VF}-2.511 . \mathrm{T}-95.3 \mathrm{VF}^{2}++0.01622 . \mathrm{T}^{2}$ & & \\
\hline $20^{\circ} \mathrm{C} \leq \mathrm{T} \leq 90^{\circ} \mathrm{C}$ & & \multirow[b]{2}{*}{8} \\
\hline$\rho=835.34+112.5 . \mathrm{VF}-0.776 . \mathrm{T}-77.8 \mathrm{VF}^{2}$ & 92.92 & \\
\hline- & & \\
\hline$\rho=861.65+160.1 . \mathrm{VF}-3.624 . \mathrm{T}-110.2 \mathrm{VF}^{2}++0.0743 . \mathrm{T}^{2}-1.11 . \mathrm{VF} . \mathrm{T}$ & 95.65 & 9 \\
\hline $15^{\circ} \mathrm{C}$ & & \\
\hline$\rho=826.02+1.239 \mathrm{~W}+120.9 . \mathrm{VF}-81.5 . \mathrm{VF}^{2}$ & 86.13 & 10 \\
\hline
\end{tabular}


Due to interaction effects between the variables, the parameters could not be analyzed independently. The significance of the parameters in the model was obtained using statistical techniques. Fig. 9 and 10 show the contour graph of the interaction effects of the three parameters (volume fractions, testing temperatures and storage periods) for the kinematic viscosity and density respectively. The contour areas help to explain how the kinematic viscosity and density varies with a change in the experimental conditions. The number written on each contour area indicates the kinematic viscosity and density values in the specified experimental conditions. These contour plots were demonstrated that the interaction effects of all parameters were considerable.
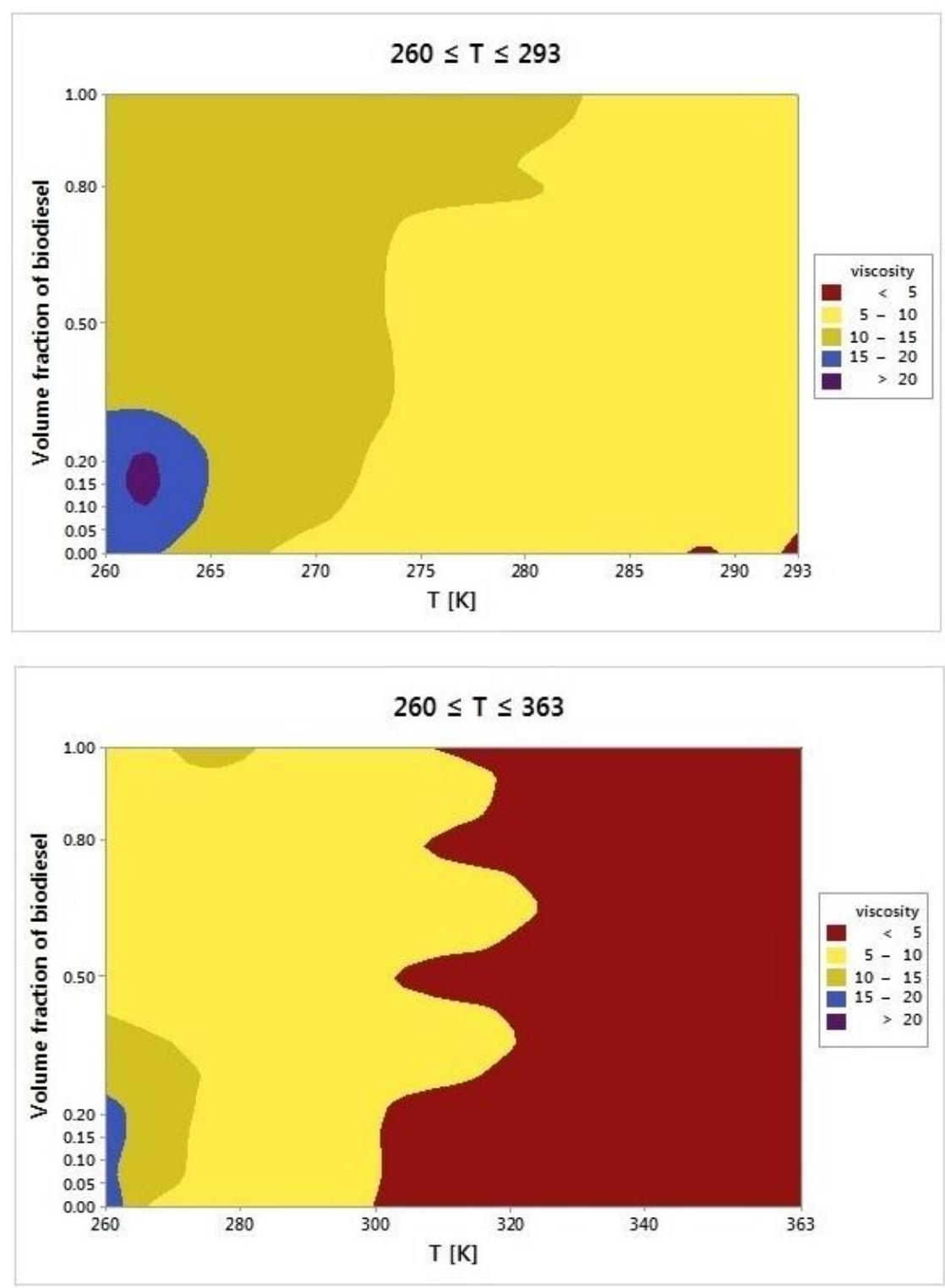

Fig. 9. Contour graph of kinematic viscosity of biodiesel blends. 

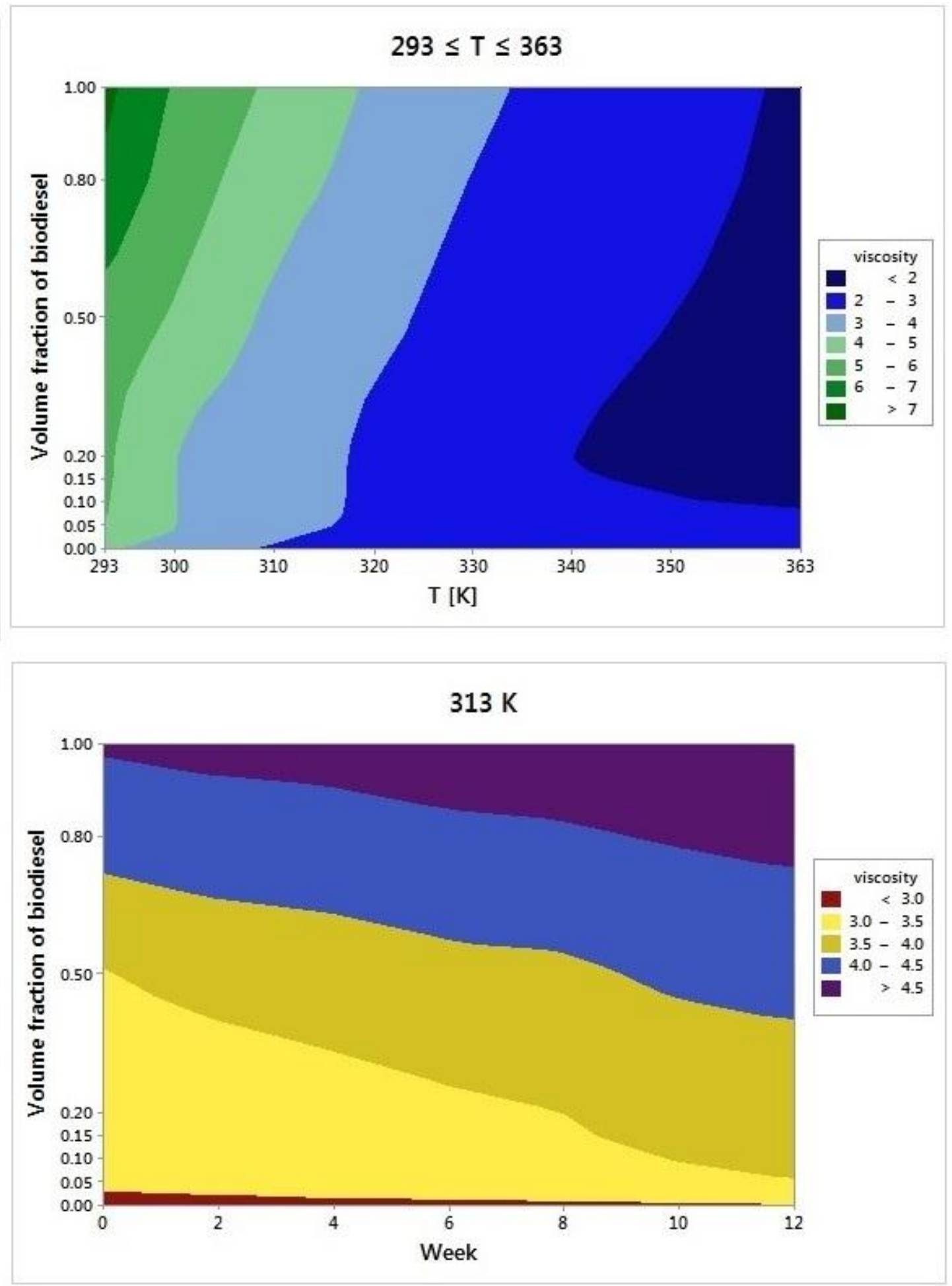

Fig. 9. Continued 

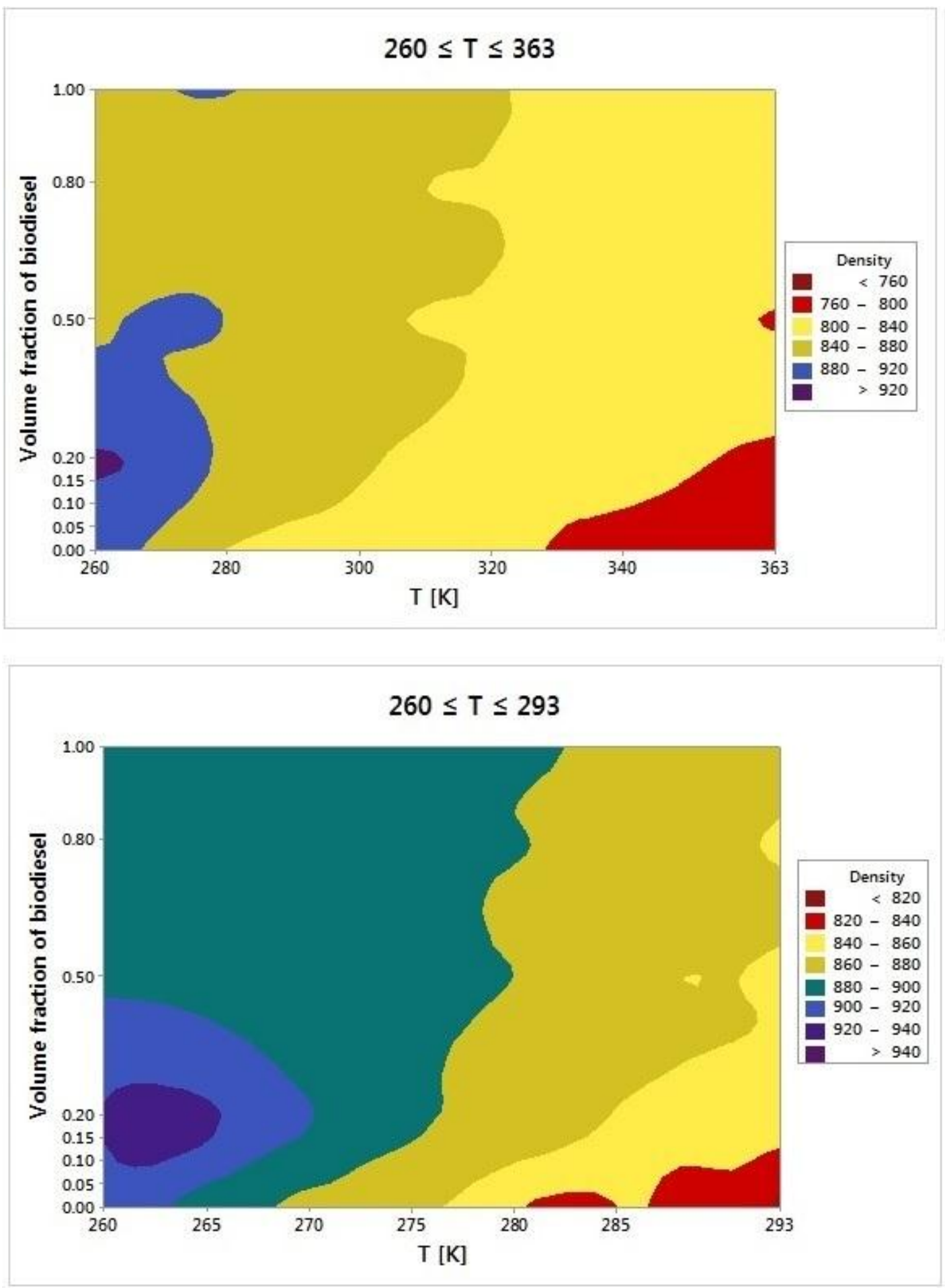

Fig. 10. Contour graph of density of biodiesel blends. 

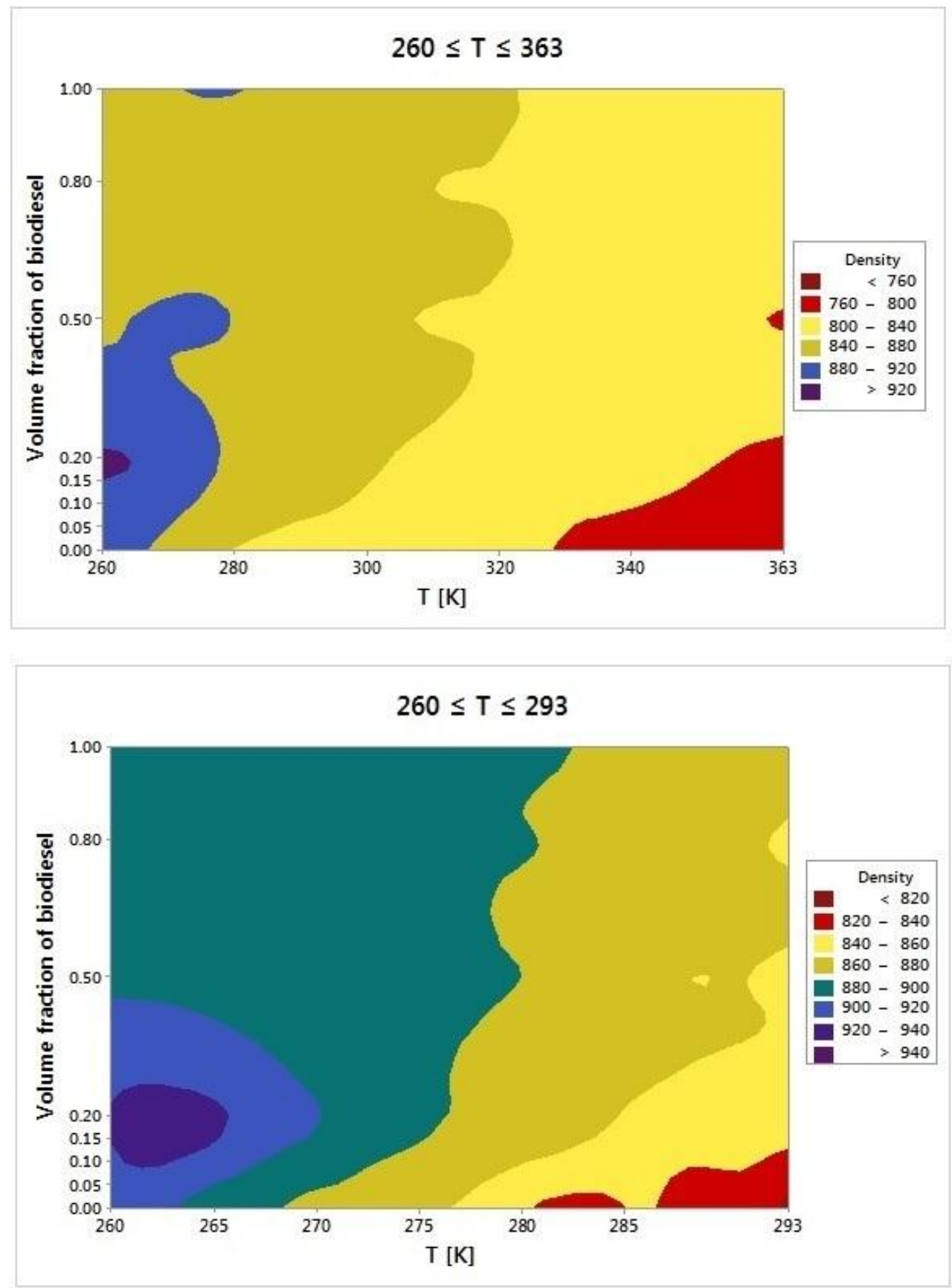

Fig. 10. Continued

\section{Conclusions and Future Works}

In this study, biodiesel was produced from a mixture of waste vegetable oils to be used as a biodiesel fuel. A study was carried out to investigate how the basic flow properties: kinematic viscosity and density for eight fuels (D100, B5, B10, B15, B20, B50, B80 and B100) change with time (week) and temperature. 
Additionally, the effects of testing temperatures and storage periods on fuel properties were experimentally examined. Based on the analysis of the results obtained, the following conclusions can be drawn:

- The experimental data showed that the viscosity and densities of diesel and all biodiesel blends decreased with an increase in temperature up to $90^{\circ} \mathrm{C}$.

- The results indicated that viscosity and densities increased for samples over long term storage.

- Over the course of 12 weeks of storage, viscosity and density of pure biodiesel least affected by storage period among other sample, as indicated by comparatively low $\mathrm{I}_{\mathrm{r}}$ values.

- The RSM method used the temperatures, biodiesel fractions and storage periods as inputs to calculate the kinematic viscosity and density of blends as outputs. Results indicate that the proposed RSM method is able to predict the accurate biodiesel density and kinematic viscosities with the overall $\mathrm{R}^{2}$ ranged between $83.30 \%$ and $96.18 \%$.

An interesting future study might involve studying other physical properties of biodiesel such as cold flow properties, acid value, oxidation stability. In addition, the effect of long term storage on properties of biodiesel blends with different diesel fuels by varying the volume fraction of biodiesel from 0 to $100 \%$ in step of $5 \%$.

\section{Acknowledgement}

The authors would like to thank the Faculty of Engineering especially the Mechanical Engineering Department at Near East University for their support and encouragement.

\section{References}

[1] Gülüm M, Bilgin A. Determination of densities and viscosities of canola oil biodiesel-diesel fuel-isopropyl alcohol blends. International Journal of Smart Grid and Clean Energy, 2017; 6(3), 165-170. 177-181

[2] Gülüm M, Bilgin A. (2017). Viscosity correlation development for corn oil biodiesel-diesel fuel blends. International Journal of Smart Grid and Clean Energy, 6(3): 165-170, 165-170.

[3] Geacai S, Iulian O, Nita I. Measurement, correlation and prediction of biodiesel blends viscosity. Fuel. 2015; 143: 268274.

[4] Wakil M, Kalam M, Masjuki H, Atabani A, Fattah IR. Influence of biodiesel blending on physicochemical properties and importance of mathematical model for predicting the properties of biodiesel blend. Energy Conversion and Management. 2015; 94: 51-67.

[5] Das M, Sarkar M, Datta A, Santra AK. Study on viscosity and surface tension properties of biodiesel-diesel blends and their effects on spray parameters for CI engines. Fuel. 2018; 220: 769-779.

[6] Meng X, Jia M, Wang T. Neural network prediction of biodiesel kinematic viscosity at 313K. Fuel. 2014; 121: 133-140.

[7] Kassem Y, Çamur H. A laboratory study of the effects of wide range temperature on the properties of biodiesel produced from various waste. Vegetable Oils. Waste and Biomass Valorization. 2017; 8(6): 1995-2007.

[8] Sarin A. Biodiesel: Production and properties. Cambridge: Royal Society of Chemistry, 2012.

[9] Wyatt VT, Hess MA, Dunn RO, Foglia TA, Haas MJ, Marmer WN. Fuel properties and nitrogen oxide emission levels of biodiesel produced from animal fats. Journal of the American Oil Chemists' Society. 2005; 82(8): 585-591.

[10] Poling BE, Prausnitz JM, O'Connell JP. The Properties of Gases and Liquids. Boston: McGraw-Hill.

[11] Knothe G, Krahl J, Van GJ. The Biodiesel Handbook, 2010.

[12] Kassem Y, Çamur H, Esenel E. Adaptive neuro-fuzzy inference system (ANFIS) and response surface methodology (RSM) prediction of biodiesel dynamic viscosity at 313 K. Procedia Computer Science. 2017; 120: 521-528.

[13] Meng X, Jia M, Wang T. Predicting biodiesel densities over a wide temperature range up to 523K. 2013. Fuel, 111,216222.

[14] Aminian A, Zarenezhad B. Accurate predicting the viscosity of biodiesels and blends using soft computing models. Renewable Energy. 2018; 120: 488-500.

[15] Kassem Y, Çamur H, Bennur KE. Adaptive Neuro-fuzzy Inference System (ANFIS) and Artificial Neural Network (ANN) for predicting the kinematic viscosity and density of biodiesel-petroleum diesel blends. American Journal of Computer Science and Technology. 2018; 8-18.

[16] Kassem Y, Çamur H. Prediction of biodiesel density for extended ranges of temperature and pressure using adaptive neuro-fuzzy inference system (ANFIS) and radial basis function (RBF). Procedia Computer Science. 2017; 120: 311-316.

[17] Khalid A, Manshoor B. Analysis of mixture formation and flame development of diesel combustion using a rapid compression machine and optical visualization technique. Applied Mechanics and Materials. 2013; 315: 293-298. 